\title{
Signature Verification using Normalized Static Features and Neural Network Classification
}

\author{
Manish Trikha, Manas Singhal, Maitreyee Dutta \\ Department of Electronics and Communication Engineering, National Institute of Technical Teacher Training and \\ Research, Chandigarh, India
}

\section{Article Info \\ Article history: \\ Received May 9, 2016 \\ Revised Aug 31, 2016 \\ Accepted Sep 13, 2016}

\section{Keyword:}

Back propagation

Neural network

Normalized static features

Offline signature verification

\begin{abstract}
Signature verification is very widely used in verification of the identity of any person. Now a days other biometric verification system has been evolved very widely like figure print, iris etc., but signature verification through computer system is still in development phase. The verification system is either through offline mode or online mode in online systems the dynamic information of a signature captured at the time the signature is made while in offline systems based on the scanned image of a signature. In this paper, a method is presented for Offline signatures Verification, for this verification system signature image is first pre-processed and converted into binary image of same size with 200x200 Pixels and then different features are extracted from the image like Eccentricity, Kurtosis, Skewness etc. and that features are used to train the neural network using back-propagation technique. For this verification system 6 different user signatures are taken to make database of the feature and results are analysed. The result demonstrate the efficiency of the proposed methodology when compared with other existing studies. The proposed algorithm gives False Acceptance Rate (FAR) as $5.05 \%$ and False Rejection rate (FRR) as $4.25 \%$.
\end{abstract}

Copyright (C) 2016 Institute of Advanced Engineering and Science. All rights reserved.

Corresponding Author:

Manish Trikha,

Department of Electronics and Communication Engineering,

National Institute of Technical Teacher Training and Research,

Chandigarh, India.

Email: manish.trikha@gmail.com

\section{INTRODUCTION}

Today there are various biometric systems implementing for identification and verification of human being like figure print, iris, vocal etc., [1-2], but handwritten signatures are considered a most important biometric for identification of genuine person, also handwritten signatures used very widely in financial institutions like banking systems. But these system are still lagging behind as depend basically on human verification i.e., only by looking at the signature, there is still no system available which provide $100 \%$ verification using digital computer. Various research carried out in the field of signature over the last two decades and a several verification and recognition models have appeared. The main aim of signature recognition is to detect forgery and also at the same time reduce rejection of genuine signature [3]. There are two different categories in handwritten signatures verification [4-5]

\section{Offline signatures verification and Online signatures verification}

In Offline signature verification system an image of a signature is captured by a digital camera or obtained by scanning a signature, which is on a paper or a document and then different features are extracted like Eccentricity, Kurtosis Skewness etc., while in case of online signatures verification used the dynamic features of the image which is taken at the time of signature is made like pressure, coordinates etc. [6-7]. 
1. Type of forgery

Forgery is to copy anyone else signature for any financial or other type of benefit. The forge related to signature can be classified into three categories such as Random forgery, Simple forgery and skilled forgery [8].

a. Random forgery: It is an unskilled attempt to copy signature from name and person don't have exact knowledge of the original signature. This type of forgery is very easy to detect through naked eyes.

b. Skilled forgery: It is most difficult to make and even detect. In this type a skilled person try to make the same signature as the original one.

This signature verification cannot be done as character recognition as signature collect as digital image.

The main procedure in verification of offline signature is first pre-processing the signature image such that noise removal, normalization of the signature image and converting signature image into binary form and then finding the different static features of the signature image like moment, skewness etc., then depending upon the these features forge signatures can easily be recognized by using one of the classification methods like Support vector machine (SVM) [9], Hidden Markov Model (HMM) and feed-forward backpropagation neural network. [10]

\section{Literature Survey}

The present research is related to a number of different areas within signature verification under offline approach, Cemil OZ in [11], reported an off-line signature recognition and verification system which is based on moment invariant method using Artificial Neural Network. Two separate neural networks are designed, one for signature recognition, and another for verification (i.e. for detecting forgery), Snehil G. jaiwal in [12], then divide the image into number of sub images and extract the features of each sub image and call it as local features, then $\mathrm{NN}$ is used as classification is used, system produces FAR 5.8\% and FRR $5.0 \%$. K.V Lakshmi in [8], static features from the image like Token length, Average value of observation, Standard deviation, Trend coefficients (slope of trend line) are used as features. Miguel A. Ferrer in [14], using rotation invariant uniform local binary pattern (LBP) and gray level co-occurrence matrices (GLMC) with MCYT offline signature database. Shashikumar D. R in [15], for this author combine the global and grid features of the signature image after pre-processing of the image, then neural network is used as a classifier system, Stéphane Armand in [16], features like Centroid, Tri-Surface, Length, Six-fold-Surface and Best-fit Feature, Indrajit Bhattacharyaa in [17], author apply pixel matching technique (PMT) for classification of forge and genuine signature, Srikanta Pal in [18], the features extracted are gradient and Zernike moment and then SVM using RBF kernel is used as classifier.

\section{RESEARCH METHOD}

In general, there are five major stages in any signature verification problem:

a. Taking signature image from digital camera: The signature is taken from digital camera and send to the computer. As shown in Figure 1.

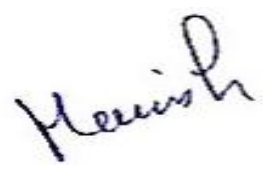

Figure 1. Sample Signature of Random User

b. Pre-processing of signature image: After image is scanned and transfer to computer it is pre-processed for removal of noise and convert the image into grey scale Figure 2.

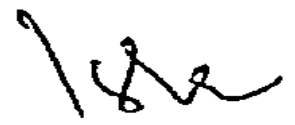

Figure 2. Pre-processed Image of Random User 
c. Covert to binary image: Image is then converted into binary image and also invert the image for more enhanced feature extraction. As shown in Figure 3

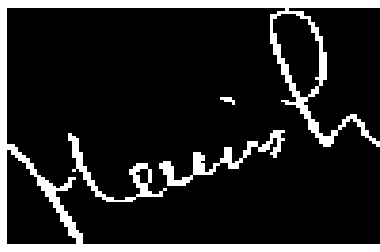

Figure 3. Binary Image of Random User

d. Feature extraction: Features extraction is a very important role to play in signature recognition and verification system, features must provide significant difference to the classifier between genuine and forge signature and at the same time should be consistent between different signatures provided by the same signor.

e. Normalization of features: After selecting the appropriate features from the signature, normalization is required as certain features magnitude are very high greater than 100 but at the same time some features have very small magnitude so, if the classification process done without normalization of features then certain features whose magnitude is large are become dominant.

f. Classification: After features extraction and normalization certain type of classification process is required in many researches different types of classification system are designed like SVM (Support Vector Machine), HMM (Hidden markov model), VQ (Vector Quantization), Backpropagation Neural Network.

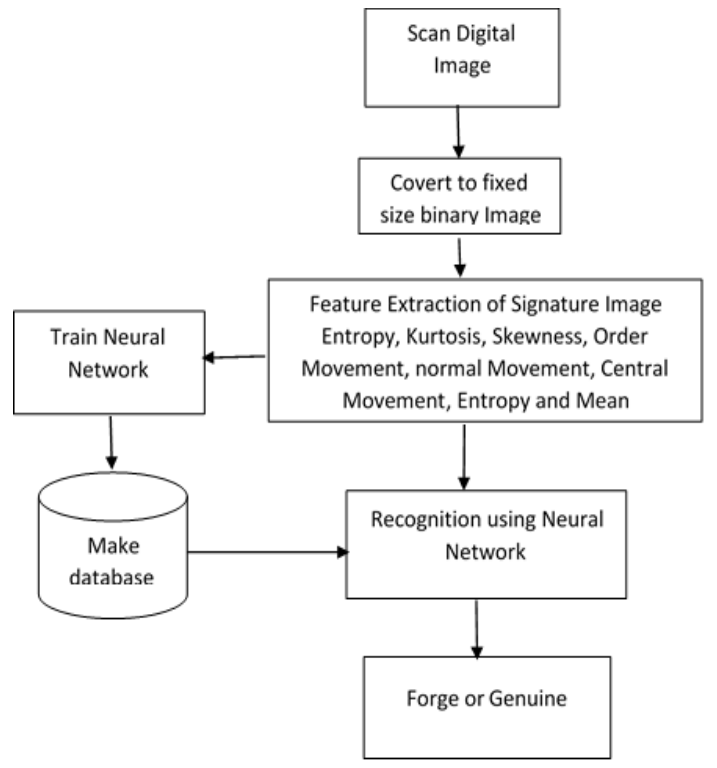

Figure 4. Flow Chart for the System

In this paper, the offline signature verification is proposed, block diagram of the whole system shown in Figure 4, system uses neural network for verification and using general back propagation optimization method for training the neural network. For the first the signature from 6 different uses are taken on blank paper and convert that signature to digital image of .jpg format and then applying pre-processing of the image for removing the noise from the image and then convert this noise free image into fixed size binary image of 200x200 pixels. 
After converting the image into binary image and then invert the image for more detail result then different features of that image are extracted from that image these features are as follows:

a. Skewness: Skewness is a measure of symmetry. A distribution, or data set, is symmetric if it looks same to the left and right of the centre point. For data Y1, Y2,.., YN, the formula for skewness is:

$$
\text { skewness }=\frac{\sum_{i=1}^{N}\left(Y_{i}-\bar{Y}\right)^{3} / N}{s^{3}}
$$

where $\bar{Y}$ the mean, $\mathrm{s}$ is is the standard deviation, and $\mathrm{N}$ is the number of data points. This formula for skewness is called Fisher-Pearson coefficient of skewness. The skewness for a normal distribution is zero, and any symmetric data should have a skewness near zero. Negative values for the skewness represent that data on which the skewness is calculated is skewed left and positive values indicate that data are skewed right.

b. Kurtosis: It is a measure of the "tailedness" of the probability distribution of any real-valued random variable. Kurtosis is a measure of shape of a probability distribution and, just like skewness, there are different ways of calculating it for a theoretical distribution and corresponding ways of estimating it from a sample of a given population. For univariate data $\mathrm{Y}_{1}, \mathrm{Y}_{2}, \ldots \ldots \mathrm{Y}_{\mathrm{N}}$, the formula for kurtosis is:

$$
\text { kurtosis }=\frac{\sum_{j=1}^{N}\left(Y_{j}-\bar{Y}\right)^{4} /_{N}}{s^{4}}
$$

where $\bar{Y}$ the mean, $\mathrm{s}$ is the standard deviation, and $\mathrm{N}$ is the number of data points

c. Moment: Moments are scalar quantities used to characterize a function and to capture its significant features. Many types of moments are there and are widely used in statistics for description of the shape of a probability density function. General moment Consider a grey-scale image $\mathrm{g}(\mathrm{x}$, $\mathrm{y})$ of width w and height $\mathrm{h}$ and pixels values in the range $0-255$. Geometric moments of a $\mathrm{p}+\mathrm{qth}$ order of $\mathrm{f}$ are given by:

$$
m_{p q}[f]=\sum_{x} \sum_{y} x^{p} y^{q} g(x, y)
$$

d. Central Moment: Central moment is a moment of a probability distribution of a random variable about the random variable's mean. The rth moment about any point $\mathrm{A}$ is called a central moment; it is the expected value of a specified integer power of the deviation of the random variable from the mean.

e. Entropy: Entropy is a measure of randomness of the pixels that can be used to characterize the texture features of the input image in digital image processing. Entropy is defined as sum $(p . * \log 2(p))$ where $p$ contains the histogram counts.

f. Mean: It is used to calculate the mean of all the white pixels in image and it can be useful as a feature of image because every signature have different lengths so total white pixels are also different.

\section{RESULTS AND ANALYSIS}

For the simulation of the proposed algorithm, six persons signatures are uses and then converted into digital image by using digital scanner, genuine signatures are used to train the neural network, then different forge signatures like random and skilled are used to test the network, before training the neural network the features are first normalized so that one feature value cannot dominate by other feature for normalization each feature is divided by its largest value to get a normalized value Table 1 show the database extracted from the normalized features of the signature image.

Each features have different values that are depend upon the user like for first user value of skewness is in 0.8 range and for user 2 it is in 0.6 range. Table 2 and Table 3 show the signature used as one of the skilled forge signature and signature used as one of the random forge signature. 
Table 1. Database of the Extracted Features from Genuine Signatures

\begin{tabular}{|c|c|c|c|c|c|c|c|c|}
\hline Feature & Image & Skewness & Kurtosis & $\begin{array}{c}\text { Order } \\
\text { Moment }\end{array}$ & $\begin{array}{c}\text { Centre } \\
\text { Moment }\end{array}$ & $\begin{array}{c}\text { Normal } \\
\text { Moment }\end{array}$ & Entropy & Mean \\
\hline User 1 & & 0.8761943 & 0.781534 & 0.5259598 & -0.9792095 & -2.0345182 & 0.6685649 & 0.5575147 \\
\hline User 1 & & 0.8667325 & 0.7660238 & 0.5062192 & -0.8894525 & -1.7843032 & 0.6771071 & 0.5667506 \\
\hline User 1 & & 0.8848492 & 0.7958688 & 0.409404 & -0.685634 & -1.4707284 & 0.6609719 & 0.5482788 \\
\hline User 1 & & 0.8350624 & 0.7153343 & 0.4689645 & -0.8165581 & -1.453937 & 0.70653 & 0.6020151 \\
\hline User 2 & & 0.6586605 & 0.4675147 & 0.9924636 & 0.6885471 & 0.5984446 & 0.9060364 & 0.861461 \\
\hline User 2 & & 0.699545 & 0.5197407 & 0.7072152 & -0.1043563 & -0.107982 & 0.8538345 & 0.7892527 \\
\hline User 2 & & 0.620566 & 0.4216824 & 0.8188501 & -0.1257713 & -0.0924689 & 0.9578588 & 0.9370277 \\
\hline User 2 & & 0.610638 & 0.4101862 & 0.7545443 & -0.2862677 & -0.2013805 & 0.9720957 & 0.9580185 \\
\hline User 3 & & 0.598715 & 0.3966249 & 1 & 0.552718 & 0.3684955 & 0.9893698 & 0.984047 \\
\hline User 3 & & 0.6914228 & 0.5091151 & 0.9491885 & 1 & 1 & 0.8638952 & 0.8035264 \\
\hline User 3 & & 0.6191586 & 0.4200414 & 0.9063721 & 0.5878255 & 0.4295284 & 0.9599468 & 0.9395466 \\
\hline User 3 & & 0.7477948 & 0.58542 & 0.6136918 & 0.3845207 & 0.4857389 & 0.7972665 & 0.7145256 \\
\hline User 4 & & 0.9045046 & 0.8289469 & 0.4115319 & -0.0259252 & -0.0597576 & 0.6440774 & 0.5289673 \\
\hline User 4 & & 0.7351448 & 0.5677768 & 0.580308 & -0.15597 & -0.1871165 & 0.8116932 & 0.7329975 \\
\hline User 4 & & 0.8528651 & 0.7435961 & 0.41632 & -0.3082721 & -0.5871467 & 0.6898254 & 0.581864 \\
\hline User 4 & & 0.8326898 & 0.7116128 & 0.4607541 & -0.3323051 & -0.5863624 & 0.7088079 & 0.604534 \\
\hline User 5 & & 0.8751767 & 0.7798578 & 0.4576223 & -1.1901598 & -2.4635412 & 0.669514 & 0.5583543 \\
\hline User 5 & & 0.883601 & 0.7937927 & 0.4815785 & -0.9531157 & -2.0351283 & 0.6621109 & 0.549958 \\
\hline User 5 & & 0.9839605 & 0.9700697 & 0.3457927 & -1.1950302 & -3.6426281 & 0.5816249 & 0.4601175 \\
\hline User 5 & & 1.0000009 & 1 & 0.3571886 & -0.9812911 & -3.1588733 & 0.5702354 & 0.4475231 \\
\hline User 6 & & 0.6052981 & 0.4040795 & 0.7184442 & -0.1241574 & -0.0852642 & 0.9796887 & 0.9697733 \\
\hline User 6 & & 0.5914234 & 0.3884632 & 0.7473421 & -0.0529295 & -0.0341347 & 1 & 1 \\
\hline User 6 & & 0.6185572 & 0.4193414 & 0.7708803 & -0.0374599 & -0.0273078 & 0.9607062 & 0.9412259 \\
\hline User 6 & & 0.6281024 & 0.4305331 & 0.6041251 & -0.5521046 & -0.4197557 & 0.9474184 & 0.9210747 \\
\hline
\end{tabular}

Table 2. Signature used as one of the Skilled Forge Signature

\begin{tabular}{|c|c|c|c|c|c|c|c|c|}
\hline Feature & Image & Skewness & Kurtosis & $\begin{array}{c}\text { Order } \\
\text { Moment }\end{array}$ & $\begin{array}{c}\text { Centre } \\
\text { Moment }\end{array}$ & $\begin{array}{l}\text { Normal } \\
\text { Moment }\end{array}$ & Entropy & Mean \\
\hline User 1 & & 0.677258 & 0.490885 & 0.826804 & -0.59709 & -0.56213 & 0.881739 & 0.827876 \\
\hline User 2 & & 1.010681 & 1.020172 & 0.365609 & -0.13308 & -0.44419 & 0.562642 & 0.439966 \\
\hline User 3 & & 0.621172 & 0.422391 & 0.540857 & -0.47321 & -0.3489 & 0.957099 & 0.935348 \\
\hline User 4 & & 0.846774 & 0.733864 & 0.508472 & 0.020206 & 0.037476 & 0.69552 & 0.588581 \\
\hline User 5 & & 0.86873 & 0.76928 & 0.415106 & -1.45106 & -2.93268 & 0.675399 & 0.565071 \\
\hline User 6 & & 0.539256 & 0.33298 & 0.899825 & -0.1576 & -0.07989 & 1.080676 & 1.128463 \\
\hline
\end{tabular}


Table 3. Signature Used as one of the Random Forge Signature

\begin{tabular}{|c|c|c|c|c|c|c|c|c|}
\hline Feature & Image & Skewness & Kurtosis & $\begin{array}{c}\text { Order } \\
\text { Moment }\end{array}$ & $\begin{array}{c}\text { Centre } \\
\text { Moment }\end{array}$ & $\begin{array}{l}\text { Normal } \\
\text { Moment }\end{array}$ & Entropy & Mean \\
\hline User 1 & Marish & 0.545644 & 0.339506 & 1.296613 & 0.791355 & 0.413394 & 1.070425 & 1.111671 \\
\hline User 2 & Devesh & 0.600045 & 0.398118 & 1.148345 & 0.445564 & 0.298933 & 0.987472 & 0.980688 \\
\hline User 3 & Isha & 1.140006 & 1.281808 & 0.220788 & 0.105056 & 0.53105 & 0.481967 & 0.356843 \\
\hline User 4 & Mansi & 1.282434 & 1.606274 & 0.301644 & 0.085604 & 0.657421 & 0.411162 & 0.289673 \\
\hline User 5 & Mansiligh & 1.050595 & 1.097565 & 0.345962 & 0.188603 & 0.718428 & 0.535687 & 0.411419 \\
\hline User 6 & Mudgha & 0.910455 & 0.839118 & 0.74908 & 0.09553 & 0.224854 & 0.639142 & 0.523929 \\
\hline
\end{tabular}

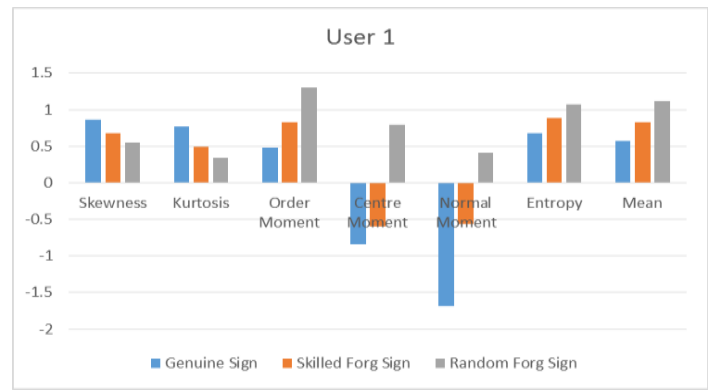

Figure 5. User 1 Signature Features Comparison

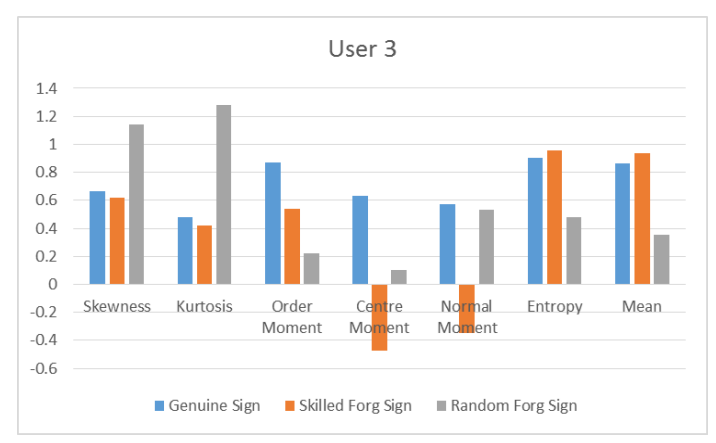

Figure 7. User 3 Signature Features Comparison

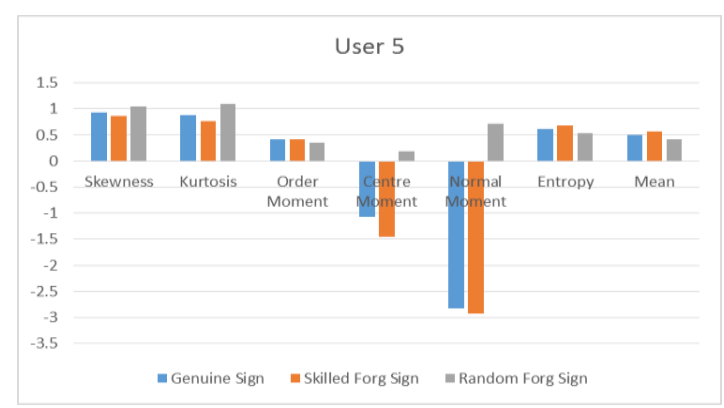

Figure 9. User 5 Signature Features Comparison

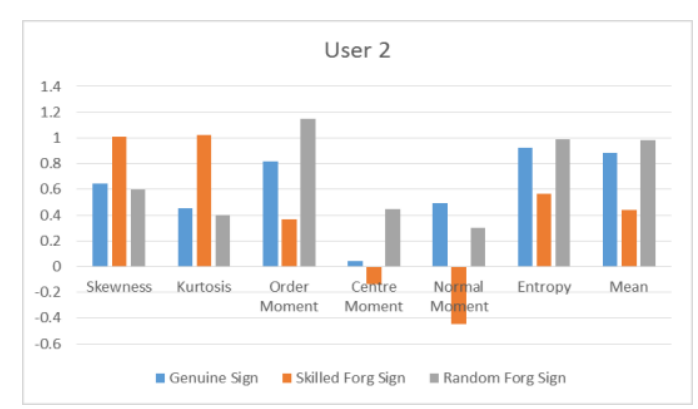

Figure 6. User 2 Signature Features Comparison

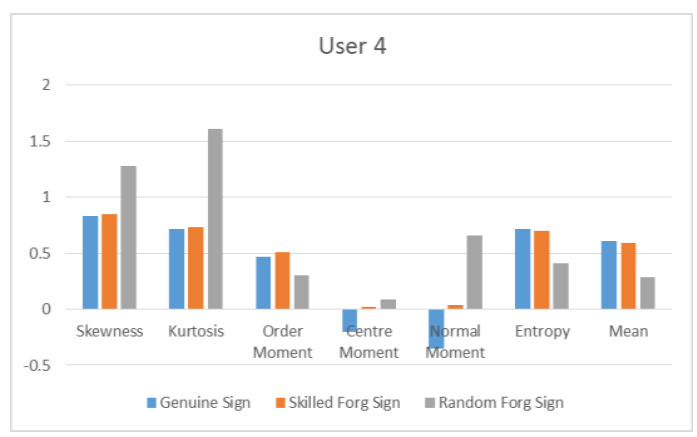

Figure 8. User 4 Signature Features Comparison

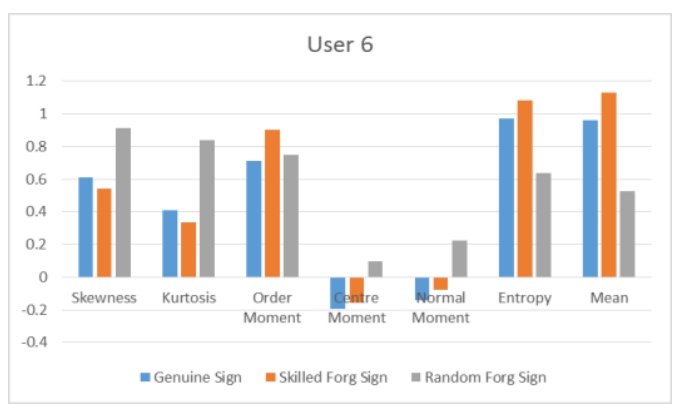

Figure 10. User 6 Signature Features Comparison 
Figure 5-10 shows that each genuine signature have different features values when compared with different forge signature types i.e., skilled and random forge. So it verifies that these features can varies if that signature is trying to be forged and can be easily identified with good classification system in this paper neural network is used a classifier.

The neural network is train for the classification and verification of the signature, for this nnstart tool of MATLAB is used and then pattern recognition application is used to recognize the signature. Table 4 shows the value of FAR (False rejection Rate) and FRR (False Acceptance Rate) comparison of the proposed system with the existing systems.

Table 4. Performance Analysis of Proposed System with Existing System

\begin{tabular}{lll}
\hline System & FAR & FRR \\
\hline Proposed System & $5.05 \%$ & $4.25 \%$. \\
{$[8]$} & $12 \%$ & $8 \%$ \\
{$[12]$} & $5.8 \%$ & $5.0 \%$ \\
{$[21]$} & $11 \%$ & $2 \%$ \\
\hline
\end{tabular}

\section{CONCLUSION}

Signature is widely used as means of personal recognition and verification process; and neural network is the verification system which is based on human brain approach for pattern recognition, so when neural network is used to verification of hand written signature then the efficiency of the verification system increases by a great amount. In this paper the Offline signatures Verification system is used for the verification of signature image. In this approach a signature image is first pre-processed for the removal of noise and converted into binary image of 200x200 Pixels and then the features, like Eccentricity, Kurtosis, Skewness etc., are extracted from the image and then normalization of the features are done and after that these normalized features are used to train the neural network using back-propagation technique. The proposed algorithm as shown in Table 4 provide more robust verification system when compared to other methods and this improvement is mainly due to normalization of the features before used in classification process which prevent the dominance of certain features over others.

\section{ACKNOWLEDGEMENTS}

The author would like to thank Director, National Institute of Technical Teacher Training and Research, Chandigarh, India for their constant support and inspiration throughout this research work.

\section{REFERENCES}

[1] Jain, A., Ross, A. and Prabhakar, S., "An Introduction to biometric recognition”, IEEE Transactions on Circuit and Systems for Video Technology, 14(1), pp. 4-20 (2004).

[2] Jungpil Shin and Tetsuya Takanashi, "Online-Signature Verification based on Pen Inclination and Pressure Information", International Journal of Electrical and Computer Engineering (IJECE), vol. 2, No.4, pp. 441-446, August 2012.

[3] A. Fallah, M. Jamaati and A. Soleamani, "A new online signature verification system based on combining Mellin transform, MFCC and neural network", Science Direct Journal on Digital Signal Process., vol. 21, no. 2, pp. 404416, 2011.

[4] Madabusi, S., Srinivas, V., Bhaskaran, S., Balasubramanian, M.:'On-line and off-line signature verification using relative slope algorithm'. Int. Workshop on Measurement Systems for Homeland Security, 2005, pp. 11-15.

[5] G. Pirlo, V. Cuccovillo, M. Diaz-Cabrera, D. Impedovo, and P. Mignone, "Multidomain verification of dynamic signatures using local stability analysis", IEEE Transactions on Human-Machine Systems, vol. 45, no. 6, pp. 805810, Dec. 2015.

[6] Gautam. S. Prakash and Shanu Sharma, "Computer vision \& fuzzy logic based offline signature verification and forgery detection", IEEE International Conference on Computational Intelligence and Computing Research (ICCIC), pp. 1-6, December, 2014.

[7] M. R Nilchiyal, R. Bte Yusof and S.E Alavi, "Statistical Online Signature Verification Using Rotation-Invariant Dynamic Descriptors", Asian Control Conference (ASCC), Kota Kinabalu, pp.1-6, 2015.

[8] K.V Lakshmi and Seema Nayak, "Off-line signature verification using neural networks", Advance Computing Conference (IACC), IEEE 3rd International Advance Computing Conference (IACC), pp.1065-1069, February, 2013. 
[9] J.F. Vargas, M.A. Ferrer, C.M. Travieso and J.B. Alonso, "Off-line signature verification based on grey level information using texture features", Science Direct Journal on Pattern Recognition, Vol. 44, Issue 2, pp.375-385, February 2011.

[10] Zhang Jian-zhong, He yong-yi and Li Jun, "Assembly Quality Prediction Based on Back-propagation Artifical Neural Network", TELKOMNIKA Indonesian Journal of Electrical Engineering, vol. 12, no. 1, pp. 179-185, January 2014.

[11] Cemil OZ, Fikret Ercal and Zafer Demir, "Signature Recognition and Verification with ANN", Chamber of Electrical Engineers (EMO -Elektrik Muhendisleri Odasi) Third International Conference on Electrical and Electronics Engineering, pp.1-5, April, 2009.

[12] Snehil G. jaiwal and Abhay R Kasetwar, "Off-line signature verification using global \& local features with neural networks", IEEE International Conference on Advanced Communication Control and Computiong Technologies (ICACCCT), pp.1525-1531, 2014.

[13] Miguel A. Ferrer, J. Francisco Vargas, Aythami Morales, and Aarón Ordóñez, "Robustness of Offline Signature Verification Based on Gray Level Features", IEEE Transactions On Information Forensics And Security, vol. 7, no. 3, pp. 966-977, June 2012.

[14] D. R. Shashikumar, K. B. Raja, R. K. Chhotaray, S. Pattanaik, "Biometric security system based on signature verification using neural networks", IEEE Conference on Computational Intelligence and Computing Research (ICCIC), Bangalore, pp. 1-6, 2010

[15] Stéphane Armand, Michael Blumenstein and Vallipuram Muthukkumarasamy, "Off-line Signature Verification using the Enhanced Modified Direction Feature and Neural-based Classification", International Joint Conference on Neural Networks Sheraton Vancouver Wall Centre Hotel, Vancouver, BC, Canada, pp. 684-691, July 16-21, 2006.

[16] Indrajit Bhattacharyaa, Prabir Ghoshb, Swarup Biswasb, "Offline Signature Verification Using Pixel Matching Technique", Elsevier (Procedia Technology 10) International Conference on Computational Intelligence: Modeling Techniques and Applications, pp. 970-977, 2013.

[17] Srikanta Pal, Umapada Pal, Michael Blumenstein, "Off-line verification technique for Hindi signatures", IEEE Transections on IET Biometrics, vol. 2, no. 4, pp. 182-190, October 2013.

[18] M.-K. Hu, "Visual pattern recognition by moment invariants," IRETrans. Information Theory, vol. 8, no. 2, pp. $179-187,1962$.

[19] Othman o-khalifa, Md. Khorshed Alam and Aisha Hassan Abdalla "An Evaluation on Offline Signature Verification using Artificial Neural Network Approach," International Conference on Computing, Electrical and Electronic Engineering (ICCEEE) (2013), pp. 368-371, 2013.

[20] Ali Karouni, Bassam Daya and Samia Bahlak, "Offline signature recognition using neural networks approach", ELSEVIER Procedia Computer Science 3, pp. 155-161, 2011.

[21] Pansare, Ashwini and Shalini Bhatia, "Handwritten Signature Verification using Neural network", International Journal of Applied Information Systems, 1(2012), pp. 44-49.

\section{BIOGRAPHIES OF AUTHORS}
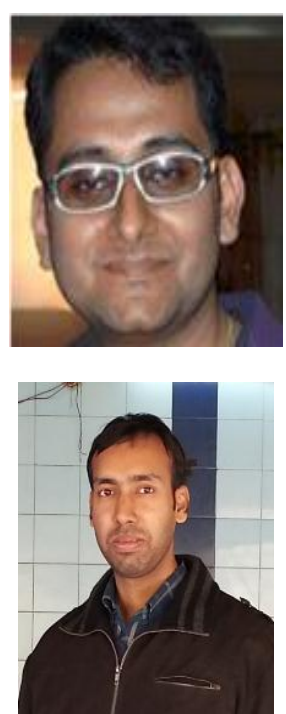

Manish Trikha received the Bachelor's degree in Electronics and Communication Engineering from Moradabad Institute of Technology (MIT), Moradabad, India in 2007, and He is pursuing Masters of Engineering degree in Electronics and Communication Engineering from National Institute of Technical Teachers' Training \& Research (NITTTR), Panjab University, and Chandigarh, India.

$\mathrm{He}$ is an Assistant Professor with the Department of Electrical Engineering, Moradabad Institute of Technology, and Moradabad, India. His current research and teaching interests are in Neural Network, Image Processing, Digital electronics and Power Electronics. He has authored 14 research publications including 9 in International Journal and 5 in International Conferences.

Manas Singhal received the Bachelor's degree in Electronics and Communication Engineering from Greater Noida Institute of Technology, UPTU, Greater Noida, and India in 2008, and He is pursuing Masters of Engineering degree in Electronics and Communication Engineering from National Institute of Technical Teachers' Training \& Research (NITTTR), Panjab University, Chandigarh, India.

He is an Assistant Professor with the Department of Electronics \& Communication Engineering, Moradabad Institute of Technology (MIT), Moradabad, India. His current research and teaching interests are in Digital Image Processing, Digital Electronics and Digital Signal Processing. He has authored 15 research publications including 10 in Journals. 


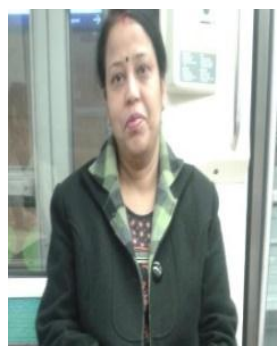

Dr. Maityree Dutta currently working as a Professor and Head of the Department, Electronics and Communication Engineering, National Institute of Technical Teacher Training and Research (NITTTR), Chandigarh, India. Her area of interest includes Digital Image Processing, Neural Network etc. She has authored various papers in Journals and Conferences. 